\title{
A World in Emergence: Cities and Regions in the 21st Century
}

\author{
by Scott, J. Allen \\ Northampton MA: Edward Elgar 2012 \\ ISBN: 978-1-78100-930-7 \\ Hardcover, \$99.95, 240 pp.
}

\author{
Reviewed by Larry S. Bourne \\ University of Toronto
}

Let me be clear: this book, although relatively short, is a tour de force. It represents the culmination of a decades-long exploration by the author, a professor of geography and public policy at the University of Southern California, Los Angeles, into the changing economies and divisions of labour within emerging global cities. The author's intention here is to develop a robust theory (or rather, a body of theories) to capture the trajectories of urbanization within contemporary capitalism. The book is elegantly written, offering a persuasive narrative in which the arguments and the prose flow smoothly from one theme to another. The reader is pulled along various lines of argument running in parallel, but ultimately these are brought back together in a concluding synthesis.

The book begins with an overview of what the author argues to be the latest phase in the global urbanization process under industrial capitalism, a period he calls third wave urbanization (as in the third industrial revolution). This phase ${ }^{1}$ is most evident in the emergence of very large global city regions that increasingly dominate the world economy. The third wave is described as something new and incomplete, but also should be seen as an elaboration and deepening of complex tendencies already evident in the earlier post-Fordist era—an era of increasingly flexible production and greater polarization in labour markets. This phase, in effect, represents an outgrowth of and a reaction to the instabilities and internal contradictions of the earlier (Fordist) period of mass production.

The author labels this newest phase of production dynamics as a cognitive-cultural economy, which serves as the foundation of an ongoing transformation of metropolitan economies around the world - that is, in turn, driven by fundamental changes in the nature and scope of capitalism itself. It is a phase characterized by the dominance of knowledge workers and firms, by creative/cultural occupations, digital and other technological innovations, and advanced financial and business services. There is of course little that is entirely new here, but Scott does a masterful job of animating the subject and bringing the arguments up to date.

The volume is divided into ten substantive chapters, including: a review of traditional theories of urbanization and agglomeration; the rise of the new economy and the resulting new global economic geography; the expanding role of emerging city regions in the global urban hierarchy; as well as the restructuring and revalorization in human capital and urban labour markets (with the resulting growth of a low-wage service underclass). Subsequent chapters explore the importance of symbolic analysts and high-order service industries; the role of the new economy in changing built form and the urban social milieu; the emergence of distinctive cultural landscapes in semi-rural and recreational areas around these cities; and finally a brief discussion of possible configurations of a future urban world. This represents an imaginative synthesis of what are often detached topics and competing lines of enquiry.

1. Readers interested in a more concise description of third wave urbanization and third wave cities, with impressive visual examples from a variety of cities and cultures, might want to look at a recent journal article by the same author: Scott, A.J. 2011. Emerging cities of the Third Wave. City 15(3-4):289-321. 
The primary focus of the book, as the brief summary above suggests, is on the economy-that is, on production systems, employment, and occupations. This is to be expected, given that the initial reference point is the evolution of industrialization under capitalism. There is passing reference to questions of population, demography, lifestyles, ethnicity and race in global cities, but these seem to be of secondary interest, and where they do appear are assumed to be largely derivative of the dominant production regime and associated labour markets. Despite the overly economic emphasis, readers from other disciplines will nonetheless find this overview of the global economy, and its diverse manifestations in particular places, to be both informative and stimulating.

The empirical base is also substantial, although examples are drawn largely from the US, and specifically from Los Angeles. This is understandable, given the author's place of residence and long-standing research agenda. The author is fully aware that this bias poses limits on generalization, but carefully acknowledges that the capitalist 'urban project' shows varying expressions in different parts of the world. At the same time, he argues that there is evidence of a convergence of urbanization processes as global capitalism expands and deepens.

Given the immense scale and scope of the coverage in this relatively compact volume, there are invariably areas in which any reviewer would like to have seen more analysis and discussion. For instance, the author does make an effort in several chapters to illustrate the impacts of macro-scale (global) urbanization processes on changes in the physical or spatial form of, and the social milieu provided within, world cities. Gentrification is one example provided. This is certainly one of the most challenging lines of enquiry addressed, and is perhaps the least successful in the book. Obviously the form of our cities is influenced by global economic trends and by the further articulation of capitalist modes of production, notably through new types of employment and contingent (and localized) divisions of labour. But these links may be tenuous at best. Urban form is also influenced by a host of other factors and processes, some of which are local and some are not. Furthermore, these forces are integrated and played out on a landscape canvas that involves numerous local actors, more or less constrained land markets, in-situ politics and public institutions, diverse cultures, and embedded historical conditions. On the other hand, it may be unreasonable to suggest adding that kind of analysis; in full detail, this would require another book of at least equal length.

Moreover, the focus on 'global' cities leaves one wondering what is happening in the vast majority of smaller cities around the world, where most people live and work. While the world's metropolises are clearly important players in the world economy, and becoming more so, they are not the only models or the only futures. Are these massive urban centres unique? Are the same trends finding similar expressions in other smaller places? Are we all doomed to be pale and undersized imitations of New York, Los Angeles, London, Paris, or Tokyo? One hopes not. On a parallel point, how global are these 'global' cities? One Japanese colleague once made the point that Tokyo is not really a world city but a very large Japanese city. In contrast, the most visible and powerful global city is obviously Washington DC. Where does it fit in the production system and in the urban hierarchy? Moreover, larger cities also tend to internalize more of their economic life, and to generate their own internal growth dynamic and synergies. There is, for example, considerable evidence that smaller places often have a higher proportion of their economic activity tied to external, if not international, sources and markets.

Finally, although the empirical base is rich, it at times seems almost flat - that is, it offers lists of only the largest global cities and lists of occupations (notably in Los Angeles). There is nothing, for example, to illustrate the outlines or actual dimensions of the global urbanization process. There is little or no empirical evidence of the flows, linkages, movements, or interactions that tie cities, regions, and countries together into a global system, driven by new industrial capitalism — or any other set of factors, for that matter. Few examples are provided of capital flows, trade, population migrations, internet connections, corporate structures, and information exchanges, examples that would confirm the expanded 'global' reach of the urbanization process under capitalism. To be fair, data on such flows and linkages are, with a few exceptions, notoriously difficult to assemble and often of poor quality. Sometimes data are restricted, due to security concerns, or due to that old capitalist tool, privatization.

Having said this, I still conclude that this is a superb book. I know of no other recent volume with a similar broad scope, internal cohesion, and argumentative rigour, as well as persuasive writing style. I strongly recommend this book to anyone with an interest in global economic transformations and the expanded role of global city regions. 